\title{
Efficacy of Protaper Universal, R Endo, Peezo Reamer on gutta percha removal: A stereomicroscopic analysis.
}

\author{
${ }^{1}$ Ramakant M Tiwari, ${ }^{2}$ Pradnya P Nikhade, ${ }^{3}$ Manoj G Chandak. \\ ${ }^{1,2,3}$ Department of Conservative Dentistry and Endodontics, Sharad Pawar Dental College, Datta Meghe \\ Institue of Medical Sciences, Wardha, India.
}

\begin{abstract}
:
Aim: The aim of this study is to compare the efficacy of Protaper Universal,R-Endo,Peezo Reamer for gutta percha removal during re-treatment by stereomicroscopic analysis.

Materials and methods: A total of 20 extracted human maxillary single rooted teeth were selected. Canal preparation was carried out by the sequential use of $K$-files up to size 30 at working length and then divided into one control group( $n=5)$ that is group I and three experimental groups II,III,IV $(n=5$ each). The experimental groups were obturated by lateral compaction of gutta percha using zinc oxide engenol sealer. During retreatment xylene severed as solvent in all groups. In group II Protaper Universal, group III R-Endo, group IV Peezo Reamer were used for gutta percha removal. Stereomicroscope was used to check the efficacy and statistically analysed.
\end{abstract}

Results: Among the three experimental groups stereomicroscopic analysis showed greatest efficacy of group III at coronal, middle and apical sections when their summation was done.

Conclusion: R-endo showed superior gutta percha removal efficacy over Protaper Universal and Peezo Reamer.

Keywords: Re-treatment, Solvent, Obturation.

\section{Introduction}

The aim of root canal retreatment is to remove the existing root canal filling material completely ,thereby allowing entire root canal system to be cleaned. The primary reason for negative outcome following root canal treatment is the persistence of bacteria within the intricacies of the root canal system. ${ }^{1}$ Besides iatrogenic factors such as inadequate canal preparation / obturation and procedural errors, other several causes are responsible for these occasional failures .

For example residual post treatment root canal infection may be inevitable in some cases due to the complexity of the root canal anatomy. ${ }^{2}$ The most commonly used root canal filling material is gutta-percha in conjunction with a sealer. The proper removal of these materials from inadequately prepared and filled canals is the major part of most root canal retreatments. Removing filling materials can be time consuming and challenging but has an important clinical impact so that instruments and irrigants may gain access to the entire root canal system, thus promoting better cleaning. ${ }^{3,4}$

There are several techniques for removing gutta-percha and sealer from filled root canals using manual files, burs, and automated devices, generally preceded by the softening of the filling material with different solvents or heat. ${ }^{5}$ However, all retreatment techniques leave residual debris in the canal walls after reinstrumentation. ${ }^{6}$

Re-infection resulting from coronal leakage is also regarded as a major contributing cause. ${ }^{7}$ In these cases ,non surgical root canal retreatment may be required to re-disinfect the canals and re-establish healthy periodontal tissues. ${ }^{8}$ The retreatment procedure requires the removal of pre existing root canal filling materials ,followed by chemo-mechanical re-instrumentation and refilling of the canals. Various instruments have been used for gutta-percha (GP) removal, including endodontic hand files, engine driven rotary files, ultrasonic tips and files, and heat carrying instruments. Chemicals are sometimes used as solvents. ${ }^{9}$ Removal of GP using hand files with or without solvents is time-consuming, especially when the filling materials are well condensed. ${ }^{10}$ Nickel-titanium (NiTi) rotary instruments have been used successfully in root canal cleaning and shaping. ${ }^{11}$

The ProTaper NiTi rotary system has been upgraded to the ProTaper Universal system, which includes shaping, finishing and retreatment instruments. The three retreatment instruments (D1, D2 and D3) are designed for removing filling materials from root canals. They have various tapers and diameters at the tip, which are size 30, 0.09 taper, size 25, 0.08 taper and size 20, 0.07 taper. The full lengths of these retreatment files are $16 \mathrm{~mm}$ for D1, $18 \mathrm{~mm}$ for D2 and $22 \mathrm{~mm}$ for D3. D1, D2 and D3 are recommended to remove filling materials from the coronal, middle and apical portions of canals respectively.

The R-Endo system consists of 4 rotary files and one hand file (Re, R1, R2, R3, and Rm). Rm is only for initial penetration because all rotary files are nonend cutting. Re is used in initial bulk removal of filling 
material with $0.12 \%$ taper and $15 \mathrm{~mm}$ length. R1 is used in coronal third with $0.08 \%$ taper $15 \mathrm{~mm}$ length. R2 is used in middle third with $0.06 \%$ taper and $19 \mathrm{~mm}$. R3 is used in apical third with $0.04 \%$ taper and $23 \mathrm{~mm}$ length with diameter.

Peezo reamers (Largo drill) are similar to Gates Glidden drills but have parallel cutting side rather than elliptical shape.They are most often used in preparing the coronal portion of the root canal for post and core. The \# 1 Largo drill has a maximum diameter of $0.70 \mathrm{~mm}$ that increases by $0.20 \mathrm{~mm}$ for each successive size until finally reaching Largo \# 6 that has a maximum diameter of $1.70 \mathrm{~mm}$. One must be careful to use the safe ended peeso drill to prevent lateral perforation. They are available in sizes 1 to 6 .

\section{Specimen selection and preparation}

\section{Materials And Method}

Extracted human maxillary anterior teeth of similar tooth length were collected. Immediately after extraction, the teeth were kept in hydrogen peroxide solution washed under running tap water for 15 minutes; the external surfaces of all teeth were cleaned with periodontal curettes to remove the debris and soft tissues. Later they were cleaned with pumice and stored in normal saline at $40^{\circ} \mathrm{C}$ until use. (This is Occupational Safety and Health Administration guidelines and regulations for Collection, storage, sterilization, and handling of extracted teeth.) After preparing access cavities, a size $15 \mathrm{~K}$ file (Dentsply Maillefer, Ballaigues, Switzerland) was inserted into the root canal of each tooth until its tip was just visible at the apical foramen. Radiographs were taken to determine the patency of the canal.

\section{Initial root canal treatment}

With a size $15 \mathrm{~K}$ file inserted in the canal, the end-point of canal preparation and filling was established $1 \mathrm{~mm}$ from the foramen. A circumferential 'staging platform' was established near the canal orifice, ensuring a uniform working length (WL) of $15 \mathrm{~mm}$ in each tooth.Cleaning and shaping were performed using a modified step-back flare technique.The cervical third was flared with sizes 1-3 Gates Glidden drills (Dentsply Maillefer). Canal preparation was carried out by the sequential use of $\mathrm{K}$ files up to size 30 at working length, a step-back procedure in $1 \mathrm{~mm}$ increments to a file size 50 was then carried out. Upon withdrawal of each instrument, canals were irrigated alternatively with $5.25 \% \mathrm{NaOCl}$ and $17 \%$ ethylenediaminetetraacetic acid (EDTA). Canals of group II,III,IV were filled with gutta percha and zinc oxide eugenol sealer using a cold lateral compaction technique whereas group I remain unfilled. The coronal extension of root fillings was uniformly limited to the level of the staging platform. The access cavities were sealed with Cavit (DeTrey Dentsply). The quality of the root fillings was confirmed using postoperative radiographs in bucco-lingual (B-L) and mesial-distal (M-D) views. All teeth were stored at $37^{\circ} \mathrm{C}$ in a humidor for 30 days to allow complete setting of the sealer.

\section{Endodontic retreatment}

Removal of GP was performed by using one of the following techniques.

Group II-Gutta percha removal carried out with Pro-taper universal re-treatment files.

Group III-Gutta percha removal using R-Endo files.

Group IV-Gutta percha removal using Peezo reamer.

\section{Group II: ProTaper Universal retreatment files}

Root fillings were removed with ProTaper Universal retreatment instruments following the manufacturers instruction. In brief, D1, D2 and D3 instruments were sequentially used in a crown-down manner to reach the pre-established working length. They were manipulated in a brushing action .The rotational speed was set at 500 r.p.m as recommended. If the rotary instruments did not advance in the canal prior to reaching the designated depth, stainless steel $\mathrm{K}$ files were used to establish a glide path before re-introducing the rotary instruments.

\section{Group III: R-Endo files}

The retreatment was carried out with R-Endo retreatment files. First Rm hand file (K-File) was used with $1 / 4$ turn, pressure directed towards the apex to create a pathway; thus, allowing the centering and the alignment of the next instrument. Re NiTi rotary file was used 1 to $3 \mathrm{~mm}$ beyond the pulp chamber floor with circumferential filing. R1 NiTi rotary file was used to penetrate from the coronal third to the beginning of the middle third through repeated apically directed pushing actions. R2 NiTi rotary file was used from the middle third to the beginning of the apical third. R3 NiTi rotary file was used at the working length with circumferential filing action. 


\section{Group IV Peezo Reamers}

The re-treatment was carried out with peezo reamer starting with number \#1 for coronal upto \#6 for apical removal of gutta percha. Thus gutta percha removal was carried out in each sample.

\section{Evaluation}

For evaluation teeth were sectioned longitudinally using diamond discs.

Parameter evaluated was remaining gutta- percha and sealer.

The residual gutta percha and sealer were imaged on the canal walls using a stereomicroscope at $8 \mathrm{X}$ magnification.

Each canal was divided into coronal ,middle and apical thirds.

$+\quad$ denotes complete removal of gutta percha and sealer.

- $\quad$ denotes residual gutta percha or sealer.

\section{Observation tables}

\begin{tabular}{|l|l|l|l|}
\hline $\begin{array}{l}\text { Control } \\
\text { group }\end{array}$ & Coronal & Middle & Apical \\
\hline 1. & + & + & + \\
\hline 2. & + & + & + \\
\hline 3. & + & + & + \\
\hline 4. & + & + & + \\
\hline 5. & + & + & + \\
\hline
\end{tabular}

\begin{tabular}{|l|l|l|l|}
\hline $\begin{array}{l}\text { Protaper } \\
\text { universal }\end{array}$ & Coronal & Middle & Apical \\
\hline 1. & + & - & - \\
\hline 2. & - & + & + \\
\hline 3. & + & + & - \\
\hline 4. & - & - & - \\
\hline 5. & + & + & + \\
\hline
\end{tabular}

\begin{tabular}{|l|l|l|l|}
\hline R-Endo & Coronal & Middle & Apical \\
\hline 1. & + & + & + \\
\hline 2. & + & - & - \\
\hline 3. & + & - & - \\
\hline 4. & + & + & - \\
\hline 5. & + & + & + \\
\hline
\end{tabular}

\begin{tabular}{|l|l|l|l|}
\hline $\begin{array}{l}\text { Peezo } \\
\text { reamer }\end{array}$ & Coronal & Middle & Apical \\
\hline 1. & + & + & - \\
\hline 2. & - & + & - \\
\hline 3. & - & - & + \\
\hline 4. & + & - & - \\
\hline 5. & - & + & - \\
\hline
\end{tabular}

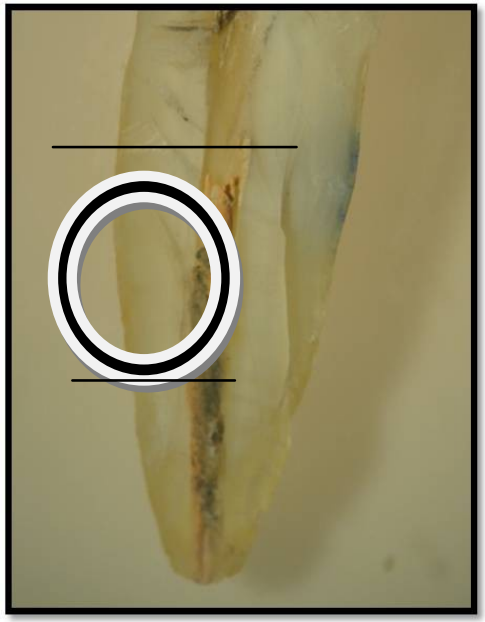

Pro-taper universal

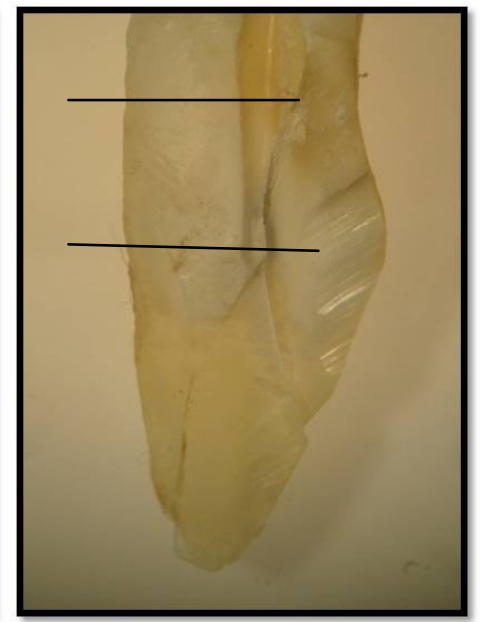

R-Endo

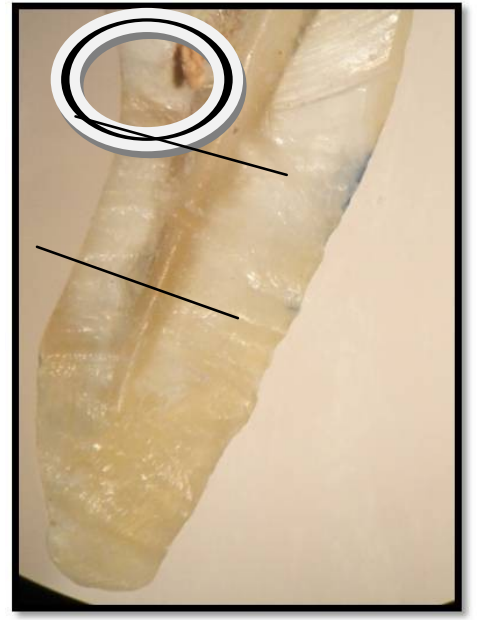

Peezo reamer

Stereomicroscopic images showing presence and absence of gutta percha and sealer with different retrearment instruments.

\section{Results}

From the above observations following results were derived- 


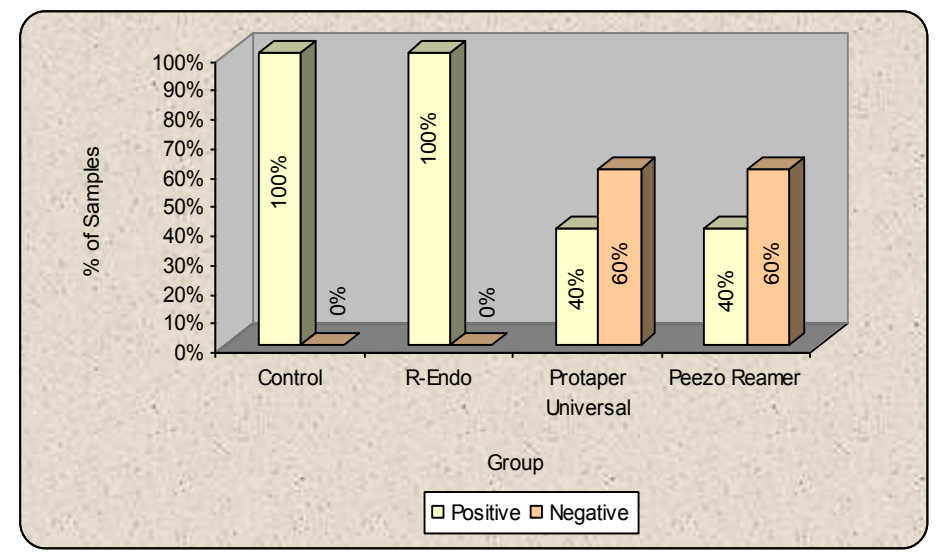

Graph 1: Comparison of Gutta Percha removal in four groups at coronal section

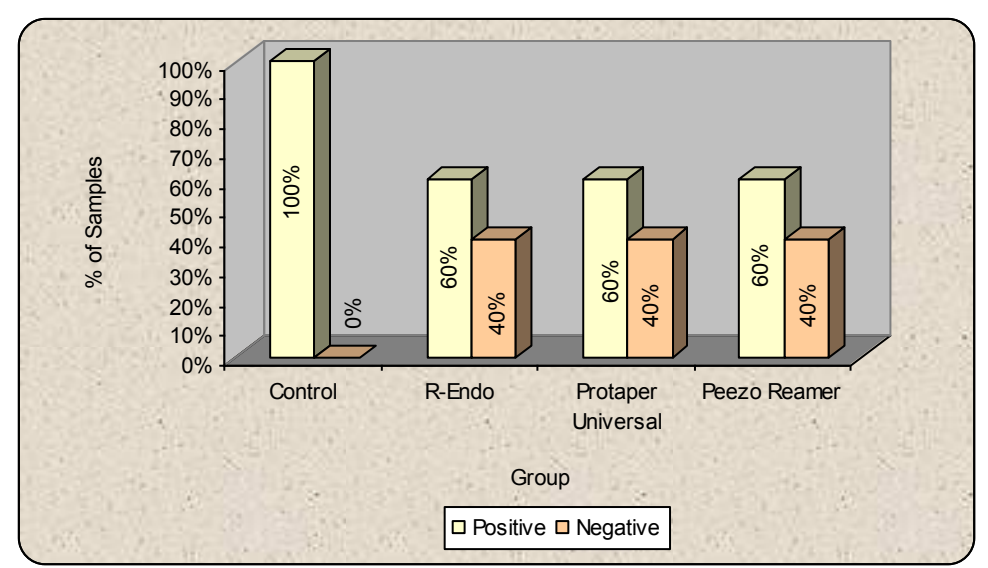

Graph 2: Comparison of Gutta Percha removal in four groups at middle section.

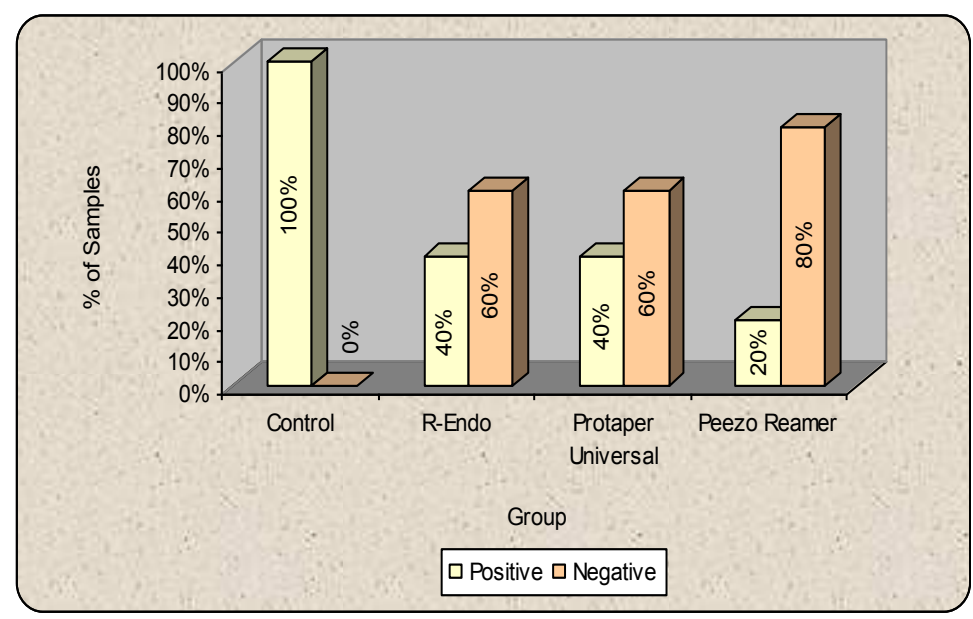

Graph 3: Comparison of Gutta Percha removal in four groups at apical section 


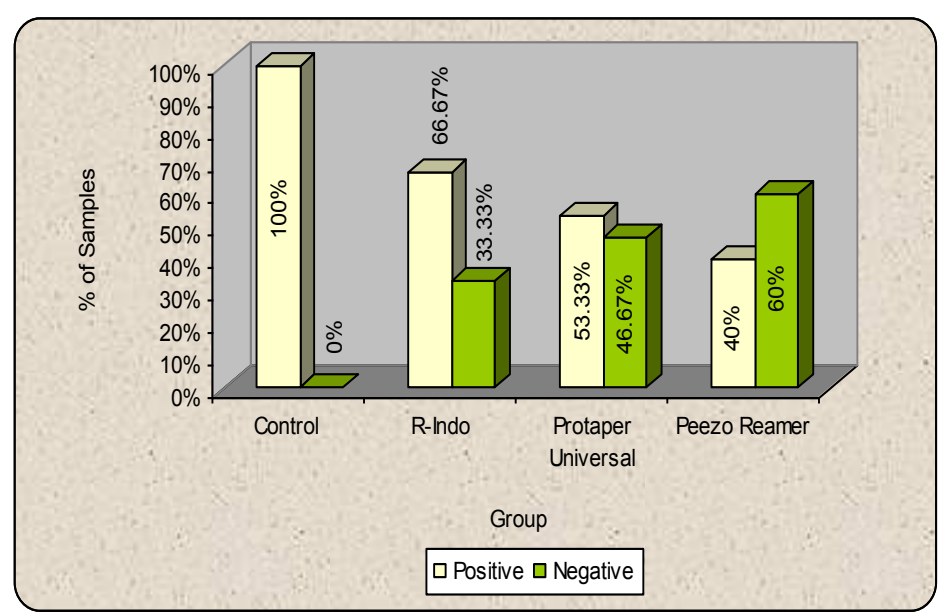

Graph 4: Comparison of Gutta Percha removal in four groups at all sections.

\section{Results-} significant.

At coronal section R-Endo is most efficient,while results of protaper universal and peezo reamer are At middle section result of all three systems are significant.

While at apical section result of R-Endo and Protaper Universal are significant and peezo reamer least efficient.

\section{Discussion}

Complete removal of pre-existing filling material from canals is a prerequisite for successful nonsurgical root canal retreatment ${ }^{12}$. This procedure can uncover residual necrotic tissues or bacteria that may be responsible for persistent periapical inflammation, and allow further cleaning and refilling of the root canal system. $^{13}$

NiTi rotary instruments have been proposed for

removing GP from root canals. ${ }^{14}$

To date, there have been few studies investigating the behaviour of retreatment instruments in nonsurgical endodontic retreatment. In the present study, the R-Endo rotary instruments left a smaller percentage of area covered by GP/sealer remnants than those treated with other techniques. The better performance of R-Endo retreatment instruments may be attributable to their design. These features may enable the retreatment instruments to cut not only GP but also the superficial layer of dentine during root filling removal. Moreover, the specific flute design and rotary motion of the R-Endo retreatment instruments tend to pull GP into the file flutes and direct it towards the orifice.

Furthermore, it is possible that the rotary movements of engine-driven files produce a certain degree of frictional heat which might plasticize GP. The plasticized GP would thus present less resistance and be easier to remove. $^{15}$

As has been shown in the literature, it was impossible to remove all traces of GP/sealer from root canals with any retreatment technique, regardless of single or combined action. This was also demonstrated in the present study, as none of the specimens was free of GP/sealer remnant under stereomicroscopic examination. The majority of remnants on the canal walls appeared to be sealer, which is consistent with other studies. ${ }^{16}$

Generally, sealer adheres well to the canal wall particularly when solvents are used. ${ }^{17}$ Therefore, effective canal re-preparation is of great importance for thorough cleaning after GP removal.Prior to the introduction of retreatment files, ProTaper rotary finishing files had been used for GP removal. This technique yielded a high-fracture incidence of $22.7 \% .{ }^{18}$

Procedural errors including instrument fracture were not noted in the present study, demonstrating the safety of retreatment instruments in endodontic retreatment. As a general rule, NiTi rotary instruments should be used with great caution. When ProTaper Universal retreatment files are used to remove GP, slight apical pressure has to be exerted for file penetration. Files should be withdrawn frequently for the removal of the debris from instrument flutes before being reintroduced in the root canal system. If the rotary instruments fail to progress along the canal path, stainless steel hand files may be used to check the resistance and establish the glide path. 


\section{Conclusion}

All techniques left GP/sealer remnants on root canal walls. The R-Endo rotary retreatment system removed GP more efficiently compared with other traditional techniques in maxillary anterior teeth.

\section{References}

[1]. Nair PN, Sjogren U, Kahnberg KE, Krey G, Sundqvist G (1990)Intraradicular bacteria and fungi in root-filled, asymptomatic human teeth with therapy-resistant periapical lesions: along-term light and electron microscopic follow-up study.Journal of Endodontics 16, 580-8.

[2]. Ayar LR, Love RM (2004) Shaping ability of ProFile and K3 rotary Ni-Ti instruments when used in a variable tip sequence in simulated curved root canals. International Endodontic Journal 37, 593-601.

[3]. Hepworth MJ, Friedman S. Treatment outcome of surgical and nonsurgical management of endodontic failures. J Can Dent Assoc 1997;63:364-71.

[4]. Gorni FGM, Gagliani MM. The outcome of endodontic retreatment:a 2-yr follow-up. J Endod 2004;30:1-4.

[5]. 5.Hulssmann M, Stotz S. Efficacy, cleaning ability and safety of different devices for gutta-percha removal in root canal retreatment. Int Endod J 1997;30:277-33.

[6]. Imura N, Zuolo ML, Kherlakian D. Comparison of endodontic retreatment of laterally condensed gutta-percha and thermafil with plastic carriers. J Endod 1993;19:609-12.

[7]. Saunders WP,Saunders EM. Coronal leakage as a cause of failure in root canal therapy: a review. Endod Dent Traumatol 1994;10:105-108.

[8]. Bergenholtz G, Lekholm U, Milthon R, Heden G, Odesjo B, Engstrom B. Retreatment of endodontic fillings. Scand J Dent Res $1979 ; 87: 217-224$.

[9]. Wilcox LR, Krell KV, Madison S, Rittman B (1987) Endodontic retreatment; evaluation of gutta-percha and sealer removal and canal reinstrumentation. Journal of Endodontics 13, 453-7.

[10]. Sae-Lim V, Rajamanickam I, Lim BK, Lee HL (2000) Effectiveness of ProFile.04 taper rotary instruments in endodontic retreatment. Journal of Endodontics 26, 100-4.

[11]. Schafer E, Lohmann D (2002) Efficiency of rotary nickeltitanium FlexMaster instruments compared with stainless steel hand Kflexofile-Part 1. Shaping ability in simulated curved canals. International Endodontic Journal 35, 505-13.

[12]. Schirrmeister JF, Wrbas KT, Schneider FH, Altenburger MJ, Hellwig E (2006c) Effectiveness of a hand file and three nickeltitanium rotary instruments for removing gutta-percha in curved root canals during retreatment. Oral Surgery, Oral Medicine, Oral Pathology, Oral Radiology, and Endodontics 101, 542-7.

[13]. Schirrmeister JF, Hermanns P, Meyer KM, Goetz F, Hellwig E(2006d) Detectability of residual Epiphany and gutta-percha after root canal retreatment using a dental operating

[14]. microscope and radiographs-an ex vivo study. International Endodontic Journal 39, 558-65.

[15]. Baratto Filho F, Ferreira EL, Fariniuk LF (2002) Efficiency of the 0.04 taper ProFile during the re-treatment of guttapercha- filled root canals. International Endodontic Journal 35, 651-4.

[16]. Betti LV, Bramante CM (2001) Quantec SC rotary instruments versus hand files for gutta-percha removal in root canal retreatment. International Endodontic Journal 34, 514-9.

[17]. Barrieshi-Nusair KM (2002) Gutta-percha retreatment: effectiveness of nickel-titanium rotary instruments versus stainless steel hand files. Journal of Endodontics 28, 454-6.

[18]. Wilcox LR (1989) Endodontic retreatment. Ultrasonics and chloroform as the final step in reinstrumentation. Journal of Endodontics 15, 125-8.

[19]. Hulsmann M, Bluhm V (2004) Efficacy, cleaning ability and safety of different rotary NiTi instruments in root canal retreatment. International Endodontic Journal 37, 468-76. 